\title{
The clinical estimation of liver size: a comparison of techniques and an analysis of the source of error
}

\author{
STEPHEN SULLIVAN， NEVILLE KRASNER， ROGER WILLIAMS
}

British Medical fournal, 1976, 2, 1042-1043

\section{Summary}

The clinical estimation of liver size using radioisotope scintiscans as a standard of reference has been shown to be very inaccurate. The main source of error is in the location of the upper border of the liver. Physical characteristics of the patient influence this measurement.

\section{Introduction}

The accurate assessment of liver size is an important and often difficult part of the clinical examination of many patients. The distance of the liver edge below the costal margin correlates poorly with its actual size, and the location of the upper border of the liver as determined by percussion must be made at the same time. ${ }^{1-3}$ This study compares different techniques for the estimation of liver size and explores some possible sources of error.

\section{Methods}

The study was performed in two parts. In the first, 50 consecutive patients were examined within three days of having technetium- $99^{m}$ sulphur colloid liver scintiscans. The scans were set up with the peak count rate over the liver. All patients were examined by a single observer without knowledge of the scintiscan results. During quiet respiration the upper and lower borders of the liver were located in the midsternal line (MSL) and the midclavicular line (MCL). Both heavy and light percussion were used to locate the upper border, while the lower border in the MSL was located by light percussion only. The lower border in the MCL was located by light percussion, palpation, and the "scratch test." The "scratch test" was performed by placing the diaphragm of a stethoscope over the xiphisternum and auscultating the abdomen while scratching it lightly in a transverse direction and advancing the scratching finger cephalad in the right MCL. If the liver edge was below the right costal margin a change in intensity and quality of the auscultated sound was noted as the liver edge was crossed.

Subsequently these measurements were compared with the liver span in the MSL and MCL as measured from the liver scintiscan record.

In the second part of the study a further 50 patients were examined in the same way but just before scintiscanning, so that the locations of the upper and lower borders could be "spotted" on to the permanent "colour dot" scan record subsequently obtained. The differences between the borders of the liver as shown by the scan and the clinical estimates could then be easily measured.

\section{Results}

Considerable variation in liver size, with a span from 7 to $29 \mathrm{~cm}$, was found in the study. Differences between clinical and scan values

Liver Unit, King's College Hospital and Medical School, London SE5 STEPHEN SULLIVAN, MD, research fellow

NEVILLE KRASNER, MD, MRCP, research fellow (present address: Southern General Hospital, Glasgow)

ROGER WILLIAMS, MD, FRCP, director and consultant physician ranged from 0 to $9.5 \mathrm{~cm}$. The lower border of the liver was not always detectable by all methods of examination, but the first part of the study (table I) showed that the estimated clinical liver size was closest to scan size when the lower liver border was palpable. However, even then only $53 \%$ of the clinical estimates came within $2 \mathrm{~cm}$ of the size of the liver as shown by the scan. Location of the lower border by percussion or "scratch test" was even less accurate. With the exception of one case clinically estimated liver size was smaller than with scan size, and if the upper border was located by heavy, rather than light, percussion the underestimation was even greater. Clinical determination of liver span in the MSL by percussion was even less accurate.

TABLE I-Difference between liver size as determined by physical examination and liver scan

\begin{tabular}{|c|c|c|c|c|c|}
\hline \multirow[b]{2}{*}{$\begin{array}{l}\text { Difference } \\
\text { between scan } \\
\text { size and } \\
\text { clinical size }\end{array}$} & \multicolumn{5}{|c|}{ Method of examination and location } \\
\hline & $\begin{array}{c}\text { MSL } \\
\text { percussion } \\
\mathbf{n}=50\end{array}$ & $\underset{\substack{\text { percussion } \\
n=47}}{\mid}$ & $\begin{array}{c}\text { MCL } \\
\text { scratch } \\
n=36\end{array}$ & $\begin{array}{c}\text { MCL } \\
\text { palpation } \\
n=32\end{array}$ & $\begin{array}{c}\text { Peternel et } a l^{5} \\
\text { palpation }+ \\
\text { percussion } \\
n=43\end{array}$ \\
\hline $\begin{array}{l}\lessgtr 1 \mathrm{~cm} \\
\leqslant 2 \mathrm{~cm} \\
\leqslant 3 \mathrm{~cm}\end{array}$ & $\begin{array}{r}7(14 \%) \\
11(22 \%) \\
23(46 \%)\end{array}$ & $\begin{array}{l}13(28 \%) \\
19(40 \%) \\
27(57 \%)\end{array}$ & $\begin{array}{r}8(22 \%) \\
15(42 \%) \\
21(58 \%)\end{array}$ & $\begin{array}{l}12(36 \%) \\
17(53 \%) \\
19(56 \%)\end{array}$ & $\begin{array}{r}7(16 \%) \\
18(42 \%) \\
25(58 \%)\end{array}$ \\
\hline
\end{tabular}

MSL $=$ Midsternal line. $\quad M C L=$ Midclavicular line.

There was an agreement within $2 \mathrm{~cm}$ of scan size in only $22 \%$ of the patients, and underestimation of liver size by greater than $2 \mathrm{~cm}$ occurred in $66 \%$ of the patients.

The results of the second part of the study, in which the clinical and scan locations of the liver borders were directly compared, showed that the major source of error was in locating the upper border of the liver (table II). In only $30 \%$ of the cases did the clinical estimate of the location of the upper border come within $2 \mathrm{~cm}$ of its location by scanning. In $66 \%$ the location of the upper border on the scan was greater than $2 \mathrm{~cm}$ (average $4 \mathrm{~cm}$ ) above the clinically estimated location. In only two cases did the scan location lie more than $2 \mathrm{~cm}$ below the clinical location. When the patients were subdivided according to build, the average error in locating the upper border was $3.8 \mathrm{~cm}$ for 39 patients with slight to medium build but $4.7 \mathrm{~cm}$ in nine obese patients. For two patients with chronic obstructive pulmonary disease and very flat diaphragms the errors were 0 and $2 \mathrm{~cm}$ respectively. A clinical estimate of lower liver border was obtained in 39 patients and with slightly better accuracy, being within $2 \mathrm{~cm}$ of its location on the scan in $54 \%$ of the cases. In those cases in which there was a difference between the two estimates of the lower border its location clinically was more frequently above the location, as shown on the scan, than below. A patient's build did not materially influence the accuracy with which the lower liver border was located clinically.

TABLE II-Difference between clinical examination and liver scan in location of liver borders

\begin{tabular}{c|c|c}
\hline $\begin{array}{c}\text { Difference between the } \\
\text { location of the border on } \\
\text { scan and physical } \\
\text { examination }\end{array}$ & $\begin{array}{c}\text { Upper border } \\
\mathrm{n}=50\end{array}$ & $\begin{array}{c}\text { Lower border } \\
\mathrm{n}=39\end{array}$ \\
\hline$\leqslant 1 \mathrm{~cm}$ & $10(20 \%)$ & $13(33 \%)$ \\
$\leqslant 2 \mathrm{~cm}$ & $15(30 \%)$ & $21(54 \%)$ \\
$\leqslant 3 \mathrm{~cm}$ & $21(42 \%)$ & $29(74 \%)$ \\
\hline
\end{tabular}

In neither part of the study was there any relationship between the accuracy with which liver size was determined clinically and the size of the liver as shown on the scan. 


\section{Discussion}

Castell et $\mathrm{al}^{4}$ have determined normal liver size by percussion and derived tables based on age, sex, height, and lean body weight. Unfortunately the accuracy of their clinical estimates was not confirmed by radiography or scintiscans. Naftalis and Leevy ${ }^{1}$ found an excellent correlation between liver size on scan and clinical estimation. However, our data and those of Peternel $e t a l^{2}$ and Halpern et $a l,{ }^{5}$ who have also used scintiscans as a reference standard, suggest that the clinical assessment of liver size is frequently very inaccurate. Blendis $e t a l^{6}$ came to a similar conclusion using abdominal radiography as a reference standard. When the data of Peternel et $a l^{2}$ are reanalysed and presented in a similar way to ours (table I) there is a striking similarity between their results and ours. They also noted the difficulty in locating the upper liver border.

This study has shown that in approximately $50 \%$ of cases liver size will be underestimated by greater than $2 \mathrm{~cm}$. Rarely will the liver size be overestimated. It is clear that the major difficulty is in percussing the upper border of the liver. The location of the upper border will be underestimated in approximately two-thirds of cases. This error will be even greater if Sherlock's suggestion ${ }^{7}$ of heavy percussion rather than light percussion is followed. While on theoretical grounds heavy percussion should be more accurate, it may be associated with a loss of fine discriminatory sense. ${ }^{8}$

In this study and in others it is assumed that liver scan size accurately reflects anatomical liver size, and, although scan size and post-mortem liver size may correlate closely, ${ }^{1}$ a number of errors may be introduced by the scanning technique. The upper border is usually sharply defined by the air-filled lung, but respiratory movements may blur it and the lower border. Similarly, if scan sensitivity is reduced, the relatively thin anterior lower border may not be readily detected, in which case the clinically located lower liver border should lie below the scanning location. In this study the converse happened more frequently.

Error in the clinical determination of liver size may arise from three sources. The method of examination or the examiner may be inaccurate, or physical characteristics of the patient may make examination difficult. Part of the error in determining liver span is undoubtedly intrinsic to the methods of physical examination. If a method of examination introduces a constant fixed error independent of observer variation or patient build, then as the liver sizes become larger the percentage error in clinical determination of their size will become smaller. No such inverse relationship was found in this study or that of Peternel et $a,^{2}{ }^{2}$ which suggests that if the methods of examination are inaccurate they introduce errors which are variable and not constant. This study did not attempt to assess the variations of a single observer, but in other studies it has not been a major factor.

The other major variable is that of physical build. Insufficient patients were examined to assess accurately the effect of build, but for those with slight to medium build the average error in locating the upper border was less than in the obese patients. Interestingly, the errors for the two patients with chronic obstructive pulmonary disease and very flat diaphragms were minimal.

We must conclude, therefore, that the clinical estimation of liver size is frequently an inaccurate determination and that any studies which use clinical liver size as a diagnostic, prognostic, or therapeutic index must be considered suspect. A very large liver may be readily detectable but lesser abnormalities will usually be underestimated, so that more reliable methods of determining liver size must be used.

SS is supported by a grant from the University of Western Ontario, London, Ontario, Canada.

\section{References}

1 Naftalis, J, and Leevy, C M, American Fournal of Digestive Diseases, 1963, 8, 236.

2 Peternel, W W, et al, American fournal of Digestive Diseases, 1966, 11, 346.

3 Riemenschneider, P A, and Whalen, J P, American fournal of Roentgenology, 1965, 94, 462.

4 Castell, D O, et al, Annals of Internal Medicine, 1969, 70, 1183.

5 Halpern, S, et al, Archives of Internal Medicine, 1974, 134, 123.

- Blendis, L M, et al, British Medical fournal, 1970, 1, 727.

7 Sherlock, S, in Diseases of the Liver and Biliary System, 5th edn, p 6. Oxford, Blackwell Scientific Publications, 1975.

${ }^{8}$ Al-Awgati, Q, Annals of Internal Medicine, 1969, 69, 868.

\section{SHORT REPORTS}

\section{Reliability of clinical techniques for detecting splenic enlargement}

The detection of an enlarged spleen is important in clinical diagnosis but seems to be subject to considerable inaccuracies. We have compared the results of palpation and percussion techniques for detecting splenic enlargement with those of hepatic scintigraphy.

\section{Patients, methods, and results}

Sixty-five consecutive unselected patients who were undergoing $99 \mathrm{~m} \mathrm{Tc}$ sulphur colloid liver scans were examined by one observer. Splenic palpation and percussion were performed on each patient in the supine and right lateral decubitus positions. On palpation the spleen was recorded only as palpable or non-palpable. In the first percussion technique ${ }^{1}$ the patient was examined in the supine position. The lowest intercostal space in the left anterior axillary was percussed and if the note was dull or became dull on deep inspiration the spleen was considered enlarged. In the second ${ }^{2}$ the patient was examined in the right lateral decubitus position. Percussion was performed along a line perpendicular to the left mid-costal margin. If dullness extended more than $8 \mathrm{~cm}$ above the costal margin the spleen was considered enlarged. The results for palpation and percussion were recorded separately and compared with the length and width of the outline of splenic radio- activity on the liver scan record. The spleen was considered enlarged on the scan if it was longer than $12 \mathrm{~cm}$ or wider than $7 \mathrm{~cm}$ or both. ${ }^{3}$

In 17 of the 65 patients the hepatic scan showed an enlarged spleen. In 12 of these patients the spleen was recorded as palpable in the supine or lateral position and, with one exception, had an increased area of splenic dullness. There was a considerable range of sizes: the tip varied from being just palpable to reaching the umbilicus. In three patients in whom the spleen was not palpated increased dullness was detected on percussion. Thus only two of the enlarged spleens were not detected by any form of physical examination and in one of these, although the spleen was considerably enlarged on scan $(19.5 \times 9 \mathrm{~cm})$, examination was complicated by ascites. In the other patient the spleen was only slightly enlarged $(13 \times 5 \mathrm{~cm})$. In eight patients with no apparent splenic enlargement on scan the spleen was thought to be enlarged by percussion. In five of these cases the spleen was

Comparison of clinical methods and radioisotope scanning in detecting splenic enlargement

\begin{tabular}{|c|c|c|c|c|c|}
\hline \multirow{2}{*}{$\begin{array}{l}\text { Spleen size } \\
\text { on scan }\end{array}$} & \multirow{2}{*}{$\begin{array}{c}\text { No of } \\
\text { patients }\end{array}$} & \multirow{2}{*}{$\begin{array}{l}\text { Spleen palpable } \\
\text { in supine or } \\
\text { lateral position }\end{array}$} & \multicolumn{2}{|c|}{$\begin{array}{l}\text { Spleen enlarged } \\
\text { by percussion }\end{array}$} & \multirow{2}{*}{$\begin{array}{l}\text { Spleen enlarged } \\
\text { by palpation } \\
\text { or percussion }\end{array}$} \\
\hline & & & $\begin{array}{l}\text { Nixon } \\
\text { method }\end{array}$ & $\begin{array}{l}\text { Castell } \\
\text { method }\end{array}$ & \\
\hline $\begin{array}{l}\text { Normal } \\
\text { Enlarged }\end{array}$ & $\begin{array}{l}48 \\
17\end{array}$ & $\begin{array}{r}5 \\
12\end{array}$ & $\begin{array}{r}3 \\
10\end{array}$ & $\begin{array}{r}8 \\
14\end{array}$ & $\begin{array}{l}8(16 \cdot 6 \%) \\
15(88 \%)\end{array}$ \\
\hline
\end{tabular}

